ISSN 1870-4069

\title{
Software de estación terrena para cohetes hidropropulsados
}

\author{
Alberto Carlos Pérez Cadena, Oswaldo Morales Matamoros, \\ Diego Alfredo Padilla Pérez, Jesús Jaime Moreno Escobar \\ Instituto Politécnico Nacional, Escuela Superior de Ingeniería Mecánica y Eléctrica, \\ Centro de Desarrollo Aeroespacial, Ciudad de México, México \\ jemoreno@esimez.mx
}

\section{Earth Station Software for Hydropropelled Rockets}

\begin{abstract}
An embedded system and an earth station software were built for the acquisition and visualization of atmospheric variables, geolocation and physical magnitudes. We used arduino and web programming and websocket js, to create a point-to-point communication between the sensor system and the web application. Finally, an integrated circuit with sensors and a web application with communication was obtained through an XBee.
\end{abstract}

Keywords: earth station, water rocket, hydropropulsion, embedded system, web development.

\section{Introducción}

El Instituto Politécnico Nacional a través del Centro de Desarrollo Aeroespacial y equipos académicos miembros del comité organizador Antares Aerospace, IPN Cuauhtémoc y la Asociación Aeroespacial de ESIME Ticomán. Convocaron, a los estudiantes de todas las instituciones educativas pertenecientes a esta casa de estudios, a participar en el Concurso Politécnico de Cohetes hidropropulsados 2019 .

El concurso busco el desarrollo de un proyecto interdisciplinario, que simule sistemas aeroespaciales complejos. Por un lado, la construcción de un cohete hidropropulsado y por otro el desarrollo de un software de adquisición de datos. El software fue un aspecto técnico importante, ya que jugo el papel de una estación terrena, mediante una plataforma de adquisición y visualización de variables en tiempo real basados en módulos embebidos. Se utilizó arduino, obteniéndose un circuito integrado con sensores que median:

- Presión,

- Temperatura,

- Humedad relativa,

- Longitud y latitud mediante GPS,

- Aceleración que sufre el dispositivo en 3 ejes, 
- Altitud.

El sistema es una aplicación web con comunicación a través de un XBee. Se uso websockets con javascript, para crear una comunicación punto a punto entre el sistema de sensores y la aplicación web.

Además, se desarrolla el sistema de información bajo un pensamiento sistémico, ya que la sistémica busca crear una sinergia que conjunta soluciones parciales, encontrando sus interrelaciones con la finalidad integrar propuestas como lo son las tecnologías de información, tecnología aeroespacial y sistemas embebidos.

Actualmente, los sistemas para realizar mediciones de variables atmosféricas en estaciones terrenas utilizados en México pueden llegar a ser de alto costo económico, y el acceso al mismo se dificulta debido a que los fabricantes constituyen un mercado reducido. La tendencia actual de utilizar software y hardware de libre distribución, permite tener acceso a dispositivos relativamente sencillos, pero con capacidad de procesamiento adecuado a la implementación de diferentes instrumentos y funcionalidades. Así, hablar de estaciones terrenas y su equipamiento es un tema amplio que requiere un análisis a profundidad, por ello el presente articulo se centra en el software de interfaz de usuario, una interfaz gráfica para realizar procesos de medición de variables y al mismo tiempo un sistema portable y escalable que forma parte del módulo de censado y captura (Payload) para el cohete del concurso.

\section{Análisis del entorno de los sistemas actuales de monitoreo}

Actualmente existen sistemas de monitoreo de diferentes variables y en lo que respecta al uso de sistemas embebidos e ingeniera de software enfocado a la web hay bibliografía variada reciente y ambos son un tópico estudiado en los últimos años. En la tabla 1 se presenta tres proyectos [8], [9], [10], que involucran tecnologías similares realizados por diversos autores con la finalidad de mostrar aportes en el proceso de investigación y desarrollo de estaciones de monitoreo.

\section{Sistemas de hidropropulsión bajo un enfoque sistémico}

\subsection{Ciencia de Sistemas}

El pensamiento sistémico no busca solucionar problemas ni proponer soluciones prácticas, más bien es una metodología integradora para el tratamiento de los problemas científicos. Por lo tanto, con un enfoque de sistemas podemos resolver los problemas de un sistema mayor en vez de sistemas componentes, nos enfocamos por optimizar la eficacia del sistema total en lugar de mejorar la eficacia de sistemas cerrados [3]. 
Software de estación terrena para cohetes hidropropulsados

Tabla 1. Proyectos relacionados con el tema en cuestión.

\begin{tabular}{|c|c|c|c|c|}
\hline Identificación & Objetivo & Categoria & Instrumentos & Resultado \\
\hline $\begin{array}{l}\text { Ruiz-Ayala, D. C., Vides-Herrera, C. } \\
\text { A., \& Pardo-García, A. (2018). } \\
\text { Monitoreo de variables } \\
\text { meteorológicas a través de un } \\
\text { sistema inalámbrico de adquisición } \\
\text { de datos. Revista de investigación, } \\
\text { Desarrollo e Innovación, 8(2), 333- } \\
\text { 341. }\end{array}$ & $\begin{array}{l}\text { Desarrollar un sistema } \\
\text { para el monitoreo } \\
\text { inalámbrico de variables } \\
\text { climáticas. }\end{array}$ & $\begin{array}{l}\text { Software y } \\
\text { hardware para un } \\
\text { sistema de } \\
\text { monitorización web } \\
\text { de señales } \\
\text { industriales. }\end{array}$ & \begin{tabular}{|l|} 
Se utilizó un \\
microcontrolador \\
MSP432 encargado del \\
muestreo y \\
transmisión de las \\
señales monitoreadas \\
a una Raspberry PI
\end{tabular} & $\begin{array}{l}\text { Integracion de } \\
\text { software y harware } \\
\text { que proporcionan } \\
\text { datos en la web para } \\
\text { su tratamiento y } \\
\text { análisis. }\end{array}$ \\
\hline $\begin{array}{l}\text { Rivera, A. F. G., \& Clavijo, F. V. } \\
\text { (2016). Monitoreo De Sensores En } \\
\text { Aplicaciones Web Embebidas. In } \\
\text { International Congress of Basic } \\
\text { Sciences and Engineering CICI (No. } \\
\text { 105, pp. 4399-4402). }\end{array}$ & $\begin{array}{l}\text { Desarrollar un prototipo } \\
\text { de cámara } \\
\text { multiespectral para uso } \\
\text { en agricultura de } \\
\text { precisión. }\end{array}$ & $\begin{array}{l}\text { Monitoreo de } \\
\text { sensors; sistemas } \\
\text { embebidos; } \\
\text { servidor web; } \\
\text { ordenador de placa } \\
\text { única. }\end{array}$ & $\begin{array}{l}\text { La Raspberry Pi } 3 \text { es } \\
\text { una computadora de } \\
\text { placa reducida. } \\
\text { Procesador ARM } \\
\text { Cortex-A53 de 64-bit }\end{array}$ & $\begin{array}{l}\text { Monitoreo de } \\
\text { sensores en tiempo } \\
\text { real. La tecnología } \\
\text { websocket facilita la } \\
\text { interacción entre } \\
\text { programas }\end{array}$ \\
\hline $\begin{array}{l}\text { Pérez, P. D. (2018). Sistema } \\
\text { embebido basado en el monitoreo } \\
\text { de consumo de energía eléctrica de } \\
\text { electrodomésticos para el control } \\
\text { del gasto. Revista Tecnología } \\
\text { Digital. Vol , } 8 \text { (1), 69-80. }\end{array}$ & $\begin{array}{l}\text { Monitorear el consumo } \\
\text { de energía eléctrica y } \\
\text { compararlo con las } \\
\text { indicaciones de datos } \\
\text { técnicos especificados y } \\
\text { corroborar los } \\
\text { resultados para localizar } \\
\text { problemas de consumo } \\
\text { excesivo. }\end{array}$ & $\begin{array}{l}\text { Sensor de potencia, } \\
\text { inducción } \\
\text { electromagnética y } \\
\text { sistema embebido. }\end{array}$ & $\begin{array}{l}\text { Sensor de potencia } \\
\text { T9B032 con arduino } \\
\text { nano. ESP } 8266 \text { para } \\
\text { enviar el } \\
\text { monitoreo del sensor a } \\
\text { una página web en } \\
\text { tiempo real. }\end{array}$ & $\begin{array}{l}\text { Un medidor de } \\
\text { potencia, } \\
\text { empleando el } \\
\text { principio de } \\
\text { inducción } \\
\text { electromagnética } \\
\text { (Bobinas), enviando } \\
\text { la señal a un nano- } \\
\text { arduino, y } \\
\text { enviandolos vía web. }\end{array}$ \\
\hline
\end{tabular}

\subsection{Sistemas espaciales de bajo costo}

Existen diferentes tipos de sistemas de exploración espacial, los más comunes son los satélites, dependiendo de su aplicación, estos varían en tamaño y masa. Según [1, p. 5], los hay desde aquellos de varios metros de envergadura, como por ejemplo el Telescopio Espacial Hubble, hasta los de poco centímetros, como los empleados en los proyectos CanSat.

Los satélites miniaturizados son diseñados de la misma manera que los satélites grandes, sin embargo, dadas las limitaciones en cuanto a espacio y masa, sus misiones suelen ser mucho más especificas [1, p. 5]. Una ventaja importante es su bajo costo, corto tiempo de preparación y la simplicidad de sus diseños. A continuación, se define una tecnología aeroespacial de bajo costo muy popular, el cansat, así como las principales variables de medición.

Plataforma Cansat. El CanSat consiste en una plataforma que simula un sistema espacial, en este caso es un nanosatélite, el cual cabe en una lata de refresco de $350 \mathrm{ml}$. Los CanSat no orbitan, pero mediante su lanzamiento a través de un cohete (de hidropropulsión, por ejemplo), un globo sonda o un avión a escala de control remoto, se pueden realizar pruebas y transmitir información de telemetría mientras descienden lentamente a tierra usando un paracaídas u otro método de descenso, desarrollando así la misión para la que fueron construidos $[2$, p. 3]. 
Variables de medición. A la tarea de fabricar y colocar en órbita satélites se le llama misión. Las misiones son bastante variadas, existen retransmisores para telecomunicaciones, telescopios ópticos, satélites con cámaras, etc. A pesar de esto y por muy diferentes que las misiones sean, siempre se cuenta con lo mismos subsistemas $[1$, p. 6]. Los subsistemas típicamente utilizan componentes comerciales para su electrónica. Dentro de estos subsistemas encontramos antenas, telemetría, sistema de potencia, control térmico, propulsión, entre otros. Las variables a medir depende de cada misión. Por ejemplo, un globo sonda "normalmente incluye una cámara, sensores de temperatura, sensores de presión, células solares, etc. y están conectados a equipos de seguimiento y comunicaciones. El globo se suelta y se eleva hasta que el globo estalla (normalmente a $25 \mathrm{~km}$ de altitud). En este punto las cargas útiles regresan a la superficie a través de paracaídas, transmitiendo datos de posición vía radio" [5, p. 2].

Estación terrena. La comunicación en tiempo real se lleva a cabo por una estación terrestre, esta forma parte del sistema de telemetría, rastreo y mando. "Una estación terrena es el equipo que se encuentra en algún punto de la Tierra y establece comunicación con el satélite; puede ser fija o estar instalada en lugares móviles, ya sean terrestres, marítimos o aéreos; son utilizadas para recibir, transmitir o ambas" [13, p. 363].

El tipo de equipo encontrado en una estación terrena varía según su uso (ya sea para comunicación transcontinental o un para un satélite) y depende también de la naturaleza de la misión, pero existen similitudes en todas las estaciones. Según [6, p. 625], los elementos principales dentro de una estación terrena son: Sistema de antena, Transmisión y recepción de equipos de radiofrecuencia, Equipos de telemetría, seguimiento y comando (TT\&C), Interfaz de usuario de datos, recuperación de datos de la misión, centro de control de la estación y el reloj del sistema.

\subsection{Ingeniería de software y aplicaciones web}

La ingeniería de software, según [11], es una disciplina de la ingeniería que comprende todos los aspectos de la producción de software, desde la etapas iniciales de la especificación del sistema hasta el mantenimiento de éste después de quede se utiliza. Sin embargo, "aunque la ingeniería consiste en seleccionar el método más apropiado para un conjunto de circunstancias, un enfoque más informal y creativo de desarrollo podría ser más efectivo en algunas circunstancias. El desarrollo informal es apropiado para el desarrollo de sistemas basados en web, los cuales requieren una mezcla de técnicas de software y de diseño gratifico" $[11$, p. 6$]$.

De acuerdo con [7, p. 47], una aplicación web (web-based application) es un tipo especial de aplicación cliente/servidor y se define bajo tres niveles típicos: el nivel superior que interacciona con el usuario (el cliente web, normalmente un navegador), el nivel inferior que proporciona los datos (la base de datos) y el nivel intermedio que procesa los datos (el servidor web). Tanto el cliente como el 
servidor (el servidor web) y el protocolo mediante el que se comunican (HTTP) están estandarizados y no han de ser creados por el programador de aplicaciones.

En una aplicación web la interfaz gráfica está en el cliente web. "El cliente web es un programa con el que interacciona el usuario para solicitar a un servidor web el envío de los recursos que desea obtener mediante HTTP, explica. La parte cliente de las aplicaciones web suele estar formada por el código HTML que forma la página web más algo de código ejecutable realizado en lenguaje de script del navegador (JavaScript)" [7, p. 47]. Hoy los frameworks Frontend facilitan la creación de interfaces de usuario, ya que tienen una estructura de archivos y carpetas de código estandarizado (HTML, CSS, documentos JS, etc.), además de tener diseño responsivo.

\subsection{Sistemas embebidos}

Según $[4$, p. 2], existen varias definiciones de un sistema embebido o empotrado, aunque a grandes rasgos se puede entender como sistemas electrónicos de propósito específico programables. [4] nos da su propia definición: "Un sistema embebido es un sistema basado en microprocesador que está diseñado para controlar una función o rango de funciones y no está diseñado para ser programado por el usuario final de la misma manera que una PC".

Así mismo, un sistema embebido es un sistema de información que cumple con el objetivo de la automatización de procesos. "Un sistema de información (SI) es un conjunto de elementos o componentes insurreccionados que recopilan (ingresan), manipulan (procesan), almacenan y difunden datos e información (de salida) y proporcionan una reacción correctiva (mecanismo de retroalimentación) para cumplir un objetivo" [12, p. 10], además de ser apoyo en el proceso de toma de decisiones.

- Sensores:El funcionamiento de un sistema embebido, en términos generales consta de: Entrada (sensores y/o periféricos), Proceso (Tiempo real), Salida (respuesta, resultados, periféricos). De este modo, a los sistemas embebidos se les puede conectar varios tipos de accesorios que le dan versatilidad en distintas tareas. Sus módulos de E/S analógicas y digitales suelen emplearse para digitalizar señales analógicas procedentes de sensores, de esta manera se tiene una interacción con diversos componentes para medir o controlar objetos del mundo real. La gran mayoría de estos son de bajo costo y bajo consumo de energía.

- Microcontroladores:El componente principal de un sistema embebido es el susbsistema de procesamiento. "Los microcontroladores se pueden considerar como sistemas independientes con procesador, memoria y periféricos, de modo que en muchos casos todo lo que se necesita para usarlos dentro de un sistema integrado es agregar software" [4, p. 11]. Existen placas de desarrollo de hardware que facilitan el uso de la electrónica y programación de sistemas embebidos, ofreciendo bajo costo económico y facilidad de uso, siendo los más comunes: Arduino, Raspberry y PIC. Su principal ventaja es tener una gran comunidad de usuarios que lo respaldan. Por lo que hay disponibles multitud de tutoriales, herramientas, ejemplos de uso, librerías y hardware de E/S. 
- Comunicaciones:Para comunicar un sistema embebido existen soluciones integradas que brindan un medio inalámbrico para la interconexión y comunicación entre dispositivos, en la tabla 2 vemos las prestaciones que ofrecen módulos de transmisión inalámbrica más usados.

Tabla 2. Comparacion entre sistemas de comunicación.

\begin{tabular}{|l|l|l|l|}
\hline & Wi-Fi & Bluethooth & XBEE \\
\hline Velocidad & $\leq 50 \mathrm{Mbps}$ & $1 \mathrm{Mbps}$ & (máx) $: 250.000 \mathrm{~b} / \mathrm{s}$ \\
\hline Rango de alcance & 50 metros & 10 metros & 1200 metros (al aire libre) \\
\hline Precio & Caro & Medio & Barato \\
\hline Configuración & Compleja & Compleja & Simple \\
\hline Duración batería & Horas & Días & Años \\
\hline
\end{tabular}

Fuente: Elaboración propia.

Como se observa los módulos XBee son una tecnología que hace frente a las necesidades de bajo costo y redes de bajo consumo, con un estándar global abierto.

\section{Materiales y métodos}

En esta sección se presenta los componente de la plataforma embebida, así como el diseño de interfaz de usuario mediante el uso de tecnologías web.

\subsection{Subsistema de sensores}

La elección de los sensores se realizó en función de la misión para el cohete hidropropulsado, es decir, deberá tener módulos de censado que midan las siguientes variables: presión, temperatura, humedad critica longitud y latitud mediante GPS, aceleración en tres ejes y altitud.

Por lo tanto se obtuvieron los siguientes sensores:

Tabla 3. Modelos de sensores utilizados.

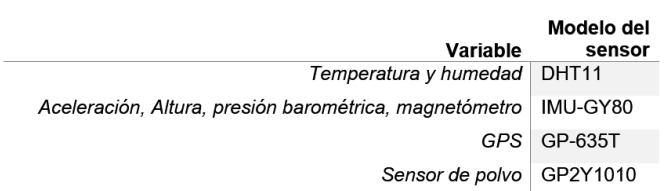

Cabe mencionar que se incluyó un sensor de calidad del aire, en específico un sensor de polvo. A continuación se hace un análisis de criterio de selección de cada uno de los sensores adquiridos. 
1. DHT11. Es un sensor digital de temperatura y humedad relativa, un dispositivo sencillo, de bajo costo y de fácil uso. A nivel de software se dispone de librerías para Arduino. En cuanto al hardware, solo es necesario conectar el pin VCC de alimentación a 3-5V, el pin GND a Tierra (0V) y el pin de datos a un pin digital en un Arduino.

2. IMU-GY80. Es un módulo muy práctico pues incluye un giroscopio, acelerómetro, brújula digital, y un sensor de presión barométrica, todo en un mismo chip. Todos los sensores individuales son accesibles a través de I2C lo que sólo necesita 4 conexiones para acceder a todos los sensores. Lo que nos ayuda a ahorrar mucho espacio.

3. GP-735T. Este GPS es delgado y tiene una antena a interna, lo que es importante pues no se tiene mucho espacio para trabajar, además tiene una sensibilidad de seguimiento muy buena y un arranque en frío de unos 27 segundos aproximadamente. Su voltaje de operación entre $3,3 \mathrm{~V}$ a 5,5 V. Lo que garantiza un buen rendimiento de batería.

4. GP2Y1010. Permite medir la concentración de polvo en el aire usando la luz infrarroja reflejada, incluso partículas humo muy finas. Tiene un consumo de corriente muy bajo (máximo $20 \mathrm{~mA}$, valor típico $11 \mathrm{~mA}$ ), además nos dará un indicador de la calidad del aire.

Elección de dispositivos de manejos de datos (Selección de antena). En la siguiente tabla vemos las prestaciones que ofrecen módulos de transmisión inalámbrica más usados.

Tabla 4. Módulos de transmisión inalámbrica más usados.

\begin{tabular}{|l|l|l|l|}
\hline & Wi-Fi & Bluethooth & \multicolumn{1}{|c|}{ XBEE S2C } \\
\hline Velocidad & $<50 \mathrm{Mbps}$ & 1 Mbps & (máx) :250.000b/s \\
\hline Rango de alcance & 50 metros & 10 metros & 1200 metros (al aire libre) \\
\hline Precio & Caro & Medio & Barato \\
\hline Configuración & Compleja & Compleja & Simple \\
\hline Duración batería & Horas & Días & Años \\
\hline
\end{tabular}

Mientras la tecnología Bluetooth está orientada a la movilidad del usuario y a eliminar el cableado entre los dispositivos de corta distancia, Xbee está orientado hacia el control remoto y la automatización, ademas el alcance en la transmisión es bastante mayor. Cabe decir, que los factores de programación de cada antena no condicionan la elección. Solo se considera nuestra prioridad un alcance amplio en el envío de datos, por tanto se decanta por el XBee S2C por ser un módulo de bajo costo y bajo consumo de energía. Con él se tiene un alcance de 1200 metros al aire libre. Esta optimizado la conexión con microcontroladores lo que ayuda con el objetivo del proyecto e incorpora la comunicación SPI (serial 
peripheral Interface) que provee un intercambio de datos con una alta velocidad entre dispositivos.

\subsection{Subsistema de potencia y diseño electrónico}

Circuito eléctrico. Al igual que con los sensores se hace un pequeño análisis de criterio de selección del sistema principal (Arduino) y subsistema de potencia.

1. Arduino Pro mini. El chip Atmega328P-AU corriendo a 16Mhz es una de las grandes ventajas de esta placa, ya que permite mantener un procesamiento óptimo a bajo consumo de energía. Se eligió por su bajo costo y diseño, ya que está pensado para aplicaciones que requieren el menor tamaño posible, además de su programación en un lenguaje de alto nivel: Processing que es similar a $\mathrm{C}++$.

2. XL6009E1. Este módulo de fuente de alimentación actúa como reguladorelevador de voltaje, tiene un rango que va de $3 \mathrm{~V}$ hasta $35 \mathrm{~V}$, es de alto rendimiento en términos de corriente de salida, proporcionando una corriente máxima de 4 amperes (con disipación de calor necesaria), es usado para aplicaciones en las que se necesita un nivel de tensión constante a la salida ante cualquier tipo de variaciones de voltaje a la entrada del módulo. De este modo, podemos conectar varios dispositivos sin una caída de voltaje.

Distribución de potencia. En la tabla 5 se presentan las características eléctricas básicas de los circuitos que conforman el sistema eléctrico:

Tabla 5. Características eléctricas básicas de los componentes de censado.

\begin{tabular}{|c|c|}
\hline Arduino & $\begin{array}{l}\text { - Voltaje de funcionamiento: } 5 \mathrm{v} \\
\text { - Voltaje de entrada: } 5-(12 \mathrm{~V} \text {, pin "RAW") } \\
\text { - Corriente máxima: } 150 \mathrm{~mA}\end{array}$ \\
\hline Xbee S2C & 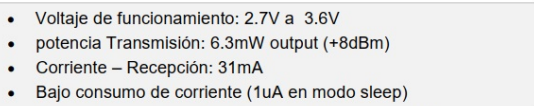 \\
\hline XL6009E1 & $\begin{array}{l}\text { Voltaje de entrada: } 3 \mathrm{~V}-32 \mathrm{~V} \text { (voltaje óptimo } 5 \mathrm{~V}-32 \mathrm{~V} \text { ) } \\
\text { - Voltaje de salida: } 5-35 \mathrm{~V} \text { Ajustable } \\
\text { Intensidad máxima de entrada: } 4 \mathrm{AA} \text { (usar disipador termico) } \\
\text { Intensidad máxima de salida: } 3 \mathrm{~A} \\
\text { Eficiencia aproximada: } 94 \% \\
\text { - Frecuencia de trabajo: } 400 \mathrm{KHz} \\
\text { Rango de temperatura trabajo: }-40 \text { a +85 grados centigrados }\end{array}$ \\
\hline DHT11 & $\begin{array}{l}\text { - Voltaje de operación: } 3.3 \text { o } 5 \mathrm{~V} \mathrm{DC} \\
\text { - Corrientes de operación: }\left(\mathrm{VCC}=5 \mathrm{~V}, \mathrm{~T}=25^{\circ} \mathrm{C}\right) \rightarrow 0.5 \text { a } 2.5 \mathrm{~mA}\end{array}$ \\
\hline GP2Y1010AU0F & $\begin{array}{l}\text { - Voltaje de funcionamiento típico: } 4.5 \mathrm{~V} \text { a } 5.5 \mathrm{~V} \text { (MAX: } 7 \mathrm{~V} \text { ) } \\
\text { - Bajo consumo de corriente (MAX: 20mA) }\end{array}$ \\
\hline GP-735 & $\begin{array}{l}\text { Corriente de Recepción: } 37 \mathrm{~mA} \\
\text { Voltaje de entrada: } 3.1 \mathrm{~V} \sim 5.5 \mathrm{~V}\end{array}$ \\
\hline IMU GY-80 & $\begin{array}{l}\text { - Voltaje: } 3.3 \mathrm{~V} \text { a } 5 \mathrm{~V} \\
\text { Derivado de la especificación para el bus } 12 \mathrm{C} \text {. Arduino EI } \\
\text { máximo absoluto para cualquier pin IO individual es de } 40 \mathrm{~mA} \text {, } \\
\text { por tanto seria el consumo para el bus I2C. }\end{array}$ \\
\hline Batería & $\begin{array}{l}\text { - Bateria de li-tio-polimero (LiPo) de } 1200 \mathrm{mAh}, 3.7 \mathrm{v} 1 \mathrm{C} \text { (1 celda), } \\
\text { con conector tipo JST hembra. }\end{array}$ \\
\hline
\end{tabular}


Utilizando la ley de ohm se explica porque el subsistema de potencia es capaz de alimentar todos los otros subsistemas, en la figura 1 se muestran las fórmulas para la demostración:

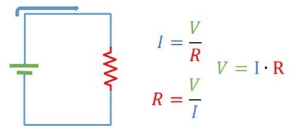

Fig. 1. Formulación matemática de la ley de ohm.

Fuente: Elaboración propia.

Las relaciones básicas en el subsistema de censado pueden verse como un circuito equivalente conectado en paralelo (figura 2). Donde Sn presentan los senadores.

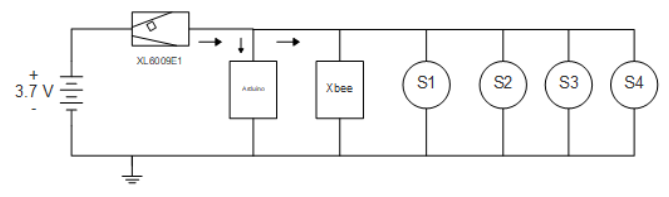

Fig. 2. Circuito equivalente conectado en paralelo. Fuente: Elaboración propia.

El circuito requiere un voltaje mínimo (lógico) de 3.3v para funcionar correctamente, sin embargo las diferentes cargas útiles pueden requerir un voltaje superior. Para cubrir esa necesidad se incluyó un voltaje secundario con regulador conmutado (dispositivo XL6009E1). Por lo tanto, el circuito se puede redefinir con una fuente de alimentación, fijando la tensión en 5 voltios, quedando el circuito anterior como el que se muestra en la figura 3:

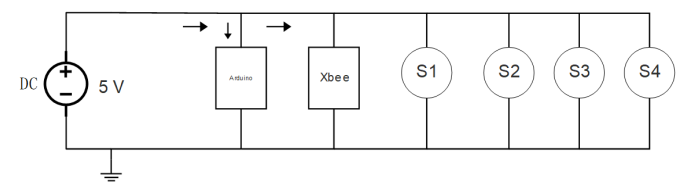

Fig. 3. Reducción del circuito equivalente conectado en paralelo. Fuente: Elaboración propia.

Como se conocen los voltajes y corrientes correspondientes a cada dispositivo y teniendo en cuenta que el circuito esta en paralelo, este se pude simplificar 
Alberto Carlos Pérez Cadena, Oswaldo Morales Matamoros, Diego Alfredo Padilla Pérez, et al.

utilizando el concepto de la resistencia equivalente. Se utilizará la formula de la figura 4, para calcular la resistencia total.

$$
R t=\frac{1}{\frac{1}{R 1}+\frac{1}{R 2}+\frac{1}{R 3}+\cdots}
$$

Fig. 4. Formula de resistencia total.

Antes de proceder con la operación, se calculan las resistencias que se tienen en cada dispositivo dentro del subsistema:

$$
\begin{gathered}
R=\frac{V}{I} \\
\text { Arduino }=\frac{5 v}{0.150 \mathrm{~A}}=\frac{100}{30} \Omega \\
\text { Xbee }=\frac{5 v}{0.031 \mathrm{~A}}=\frac{5000}{31} \Omega \\
\text { DHT11 }=\frac{5 v}{0.0025 \mathrm{~A}}=2000 \Omega \\
\text { GP2Y1010AU } \Omega=\frac{5 v}{0.020 \mathrm{~A}}=250 \Omega \\
\text { GPS }=\frac{5 v}{0.037 \mathrm{~A}}=\frac{5000}{37} \Omega \\
\text { GY80 }=\frac{5 v}{0.040 \mathrm{~A}}=125 \Omega
\end{gathered}
$$

Fig. 5. Calculo de las resistencias del subsistema.

Teniendo el valor de las resistencias, podemos calcular la resistencia equivalente, entonces:

$$
\begin{gathered}
R t=\frac{1}{\frac{3}{100}+\frac{31}{5000}+\frac{1}{2000}+\frac{1}{25}+\frac{37}{5000}+\frac{1}{25}} \Omega \\
R t=\frac{10000}{561} \Omega \approx 17.825311943
\end{gathered}
$$

Fig. 6. Resistencia equivalente.

Con estas operaciones ya es posible calcular un nuevo circuito con la ley de $\mathrm{Ohm}$. En comparación con el circuito en serie la cantidad de resistencia es menor porque se divide la corriente. De esta forma, en la figura 7 , tenemos el circuito equivalente. 
Software de estación terrena para cohetes hidropropulsados

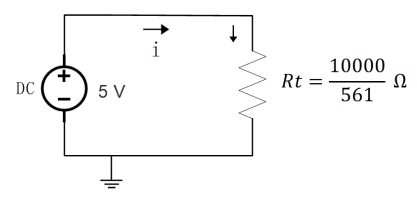

Fig. 7. Reducción del circuito equivalente conectado en paralelo. Fuente: Elaboración propia.

Con el circuito equivalente se obtiene la corriente total del circuito, usando la Ley de Ohm. Tenemos el voltaje (5V), la resistencia total (Rt), entonces la corriente total se obtiene como en la figura 8.

$$
\mathrm{I}_{t}=\frac{\mathrm{V}}{\mathbf{R}}=\frac{5 \mathrm{v}}{\frac{10000}{561}}=\frac{2805}{10000}=280.5 \mathbf{m A}
$$

Fig. 8. Corriente total.

La batería que se está utilizando nos da una corriente de $1200 \mathrm{mAh}$, con lo cual podemos calcular el tiempo de descarga de la batería en función al subsistema de sensado, entonces tenemos la resistencia equivalente (Fig. 9).

$$
\begin{gathered}
\frac{280.5 \mathrm{~mA}}{1200 \mathrm{mAh}} \approx 0.23 \mathrm{hrs} \\
\text { Si tenemos que: } \frac{1 \mathrm{~h}}{0.23 \mathrm{~h}}=\frac{60 \mathrm{~min}}{\mathrm{t}}
\end{gathered}
$$

Entonces tenemos un tiempo aproximado de

$$
t=13.8 \mathrm{~min}
$$

Fig. 9. Resistencia equivalente.

Con lo cual el subsistema que alimentamos con la batería tendría una corriente apropiadamente suministrada por un total de 14 min aproximados, sin sufrir pérdidas de energía, con lo cual para al objetivo que persigue el cohete hidropropulsado que es la medición de variables después de su lanzamiento, es cubierto por el sistema de potencia pues se satisfacen las necesidades de energía.

\subsection{Subsistema de comunicaciones y manejo de datos}

Diagrama de flujo de datos. Se muestra la representación gráfica del flujo de datos a través de un diagrama de flujo de datos o DFD (sus siglas en español e inglés) para el sistema de información que se encargara del manejo de datos y la comunicación entre el cohete y la estación terrena. 
Alberto Carlos Pérez Cadena, Oswaldo Morales Matamoros, Diego Alfredo Padilla Pérez, et al.
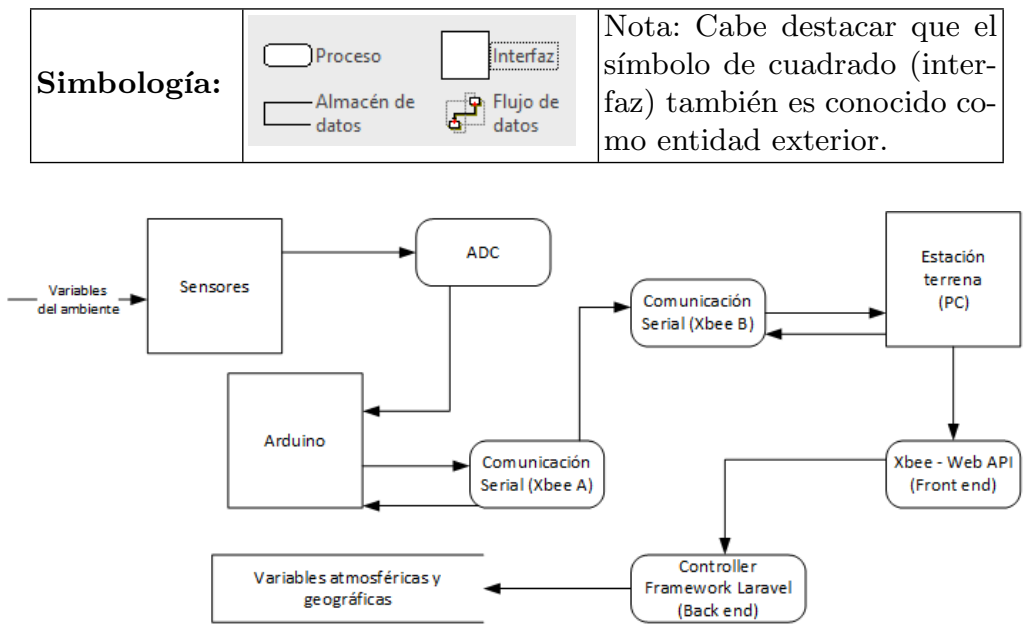

Fig. 10. Diagrama de Contexto: Nivel 0.

Fuente: Elaboración propia.

La figura 10 nos muestra las variables del entorno o ambiente que serán el insumo de entrada para nuestro sistema. Entre los sensores y el sistema Arduino existe un proceso de conversión de señal analógica a digital. Posteriormente se realiza el proceso de comunicación inalámbrica por medio de dos sistemas Xbee (A y B). Donde el Xbee A está transmitiendo los datos que te tomaron de los sensores y el Xbee B los recibe y transfiere a la computadora. La computadora actúa como un servidor remoto y se comunica con el Xbee B mediante un API en JavaScript. Teniendo los datos del lado del cliente (front end) enviadnos la información para su almacenamiento, por medio de un proceso de guardado que se realiza por un framework (back end).

\subsection{Diagrama de comunicaciones}

A grandes rasgos la comunicación es la transferencia de información con sentido desde un lugar (remitente, origen, fuente, transmisor) a otro lugar (destino, receptor), tal y como se muestra en la figura 11.

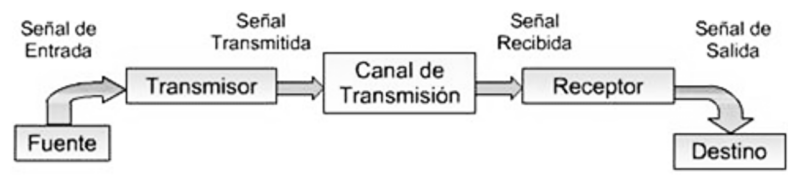

Fig. 11. Elementos básicos de un sistema de comunicaciones.

Fuente: Elaboración propia. 
Bajo la premisa anterior, el sistema de telemetría, para la misión del cohete hidropropulsado, lleva a cabo la implementación de este concepto como se muestra en la figura 12. Aquí se presentan bloques del sistema de comunicación, donde la fuente de información principal proviene del exterior y el transductor de entrada serán las señales de los sensores de entrada.

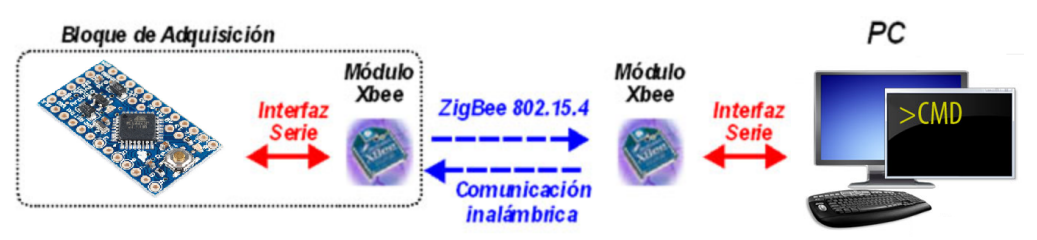

Fig. 12. Elementos básicos de un sistema de comunicaciones.

Fuente: Elaboración propia.

En este punto se puede describir la comunicación que tendrán entre cada sistema principal de la misión.

1. Concepción General del Sistema de Adquisición de Datos (Bloque de Adquisición): El Bloque de Adquisición, ubicado junto al grupo de sensores, está integrado por un microcontrolador y conectado con un módulo de comunicación inalámbrica XBee. El sistema podrá adquirir varias señales analógicas. La presencia de un microcontrolador con entradas analógicas permite digitalizar la señal analógica recibida, lo que facilitará su procesamiento y transmisión. El usuario recibirá la información del monitoreo en tiempo real o la podrá consultar cuando desee, dado que los datos adquiridos se almacenarán.

2. Comunicación inalámbrica: Los XBee está basados en el estándar ZigBee. ZigBee es una tecnología inalámbrica basada en el protocolo IEEE 802.15.4, una tecnología de red inalámbrica que opera a 2,4GHz. Desde el programa que se ejecuta en el microcontrolador se configura el módulo conectado a él, y desde la PC se configura el otro módulo con la herramienta XCTU conectado a esta. El usuario solo tiene que garantizar que el dato a transmitir esté en la salida del puerto serie del microcontrolador. Los módulos XBee se encargan de ejecutar todas las tareas necesarias para la comunicación.

3. Comunicación con el servidor: Se trabaja con sockets en Node.js, con esto tenemos un API (front end) que se utiliza para comunicarse con el módulo de radio de protocolo 802.15.4 y ZigBee. El socket no se encarga de la conexión serie en sí, pero se podrá conectar a módulos como serialport (una dependencia npm), que facilitan el acceso al puerto serial. Por otra parte, para lograr una integración entre el xbee y el servidor (en back end) se tienen dos sitios web separados capaces de comunicarse a través de un servicio web. Se tiene el primer sitio web (A) que interactúa con los datos directamente, es decir, el módulo xbee y este expone la API, para que el otro sitio web (B), bajo el framework Laravel, lo consuma. De este modo el framework 
podrá interactuar con la base datos (en un futuro) y crear los registros de los datos capturados.

\subsection{Protocolo de recepción y muestreo de datos}

En la figura 13 muestra un protocolo de recepción y muestreo de datos básico y se establece de la siguiente manera:

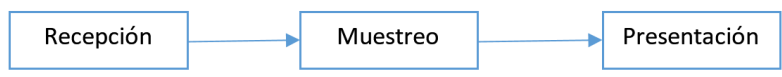

Fig. 13. El protocolo de recepción y muestreo de datos. Fuente: Elaboración propia.

- Recepción de datos. Este objetivo se cumple gracias al módulo hardware XBee, que captura señales y las pone de forma digital. Los módulos XBee tienen dos formas de trabajo: el modo transparente y el modo API. Para el proyecto se utilizó el modo transparente. Este modo está destinado principalmente a la comunicación punto a punto, donde no es necesario ningún tipo de control.

- Muestreo de datos. El objetivo es analizar las señales que se obtuvieron en la etapa anterior. Como se mencionó la comunicación es transparente, es decir, se recibe lo que se envía. En el modo transparente el dispositivo funciona como un puente Serial-Zigbee, es decir, la información que se recibe por la USART es transmitida por el aire, en una frecuencia de trabajo de $433 \mathrm{MHz}$, debido a que es una banda de libre uso.

- Presentación web de los datos muestreados. El objetivo de esta etapa es la presentación web de los datos muestreados. Este objetivo se cumplió diseñando dos servidores web que usan JavaScript y node.js.

\subsection{Diagrama de bloques de estación terrena}

A continuación, en la figura 14 se presenta el diagrama de bloques para el software de la estación terrena:

En primer lugar se tiene al módulo Xbee, este es el encargado de recibir los datos que provienen de los sensores. Este se conecta a la PC haciendo uso de Serialport-API. Se usa los sockets de JavaScript para lograr un enlace $\mathrm{UART}^{1}$. Se puede decir que se tiene un primer cliente que le hará peticiones a un servidor web.

${ }^{1}$ UART, son las siglas en inglés de Universal Asynchronous Receiver-Transmitter, en español: Transmisor-Receptor Asíncrono Universal, es el dispositivo que controla los puertos y dispositivos serie. 


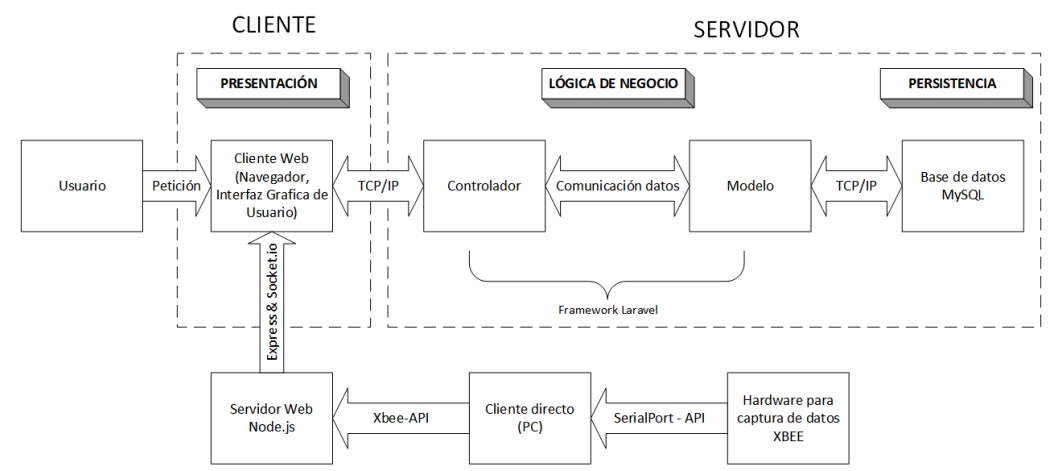

Fig. 14. Diagrama de bloques para el software de estación terrena. Fuente: Elaboración propia.

Una vez establecido el enlace UART, es preciso enviar estos datos al servidor web, el cual está alojado en una máquina remota (PC). Para lograr esto se hace uso de Node.js y express.js este último es un frawework web. El módulo Express, es el encargado de enviar el archivo HTML al cliente (el front end), cuando este realice solicitudes al portal, y así el módulo xbee-api, se dedicara a manejar el tráfico de información (las variables a monitorear) entre el cliente y el servidor.

La arquitectura utilizada para el diseño de la aplicación (el back end) está dividida en 3 capas (Presentación, Lógica del Negocio y Datos) y 2 niveles (Cliente y Servidor). La capa de presentación posee la interfaz gráfica de usuario. La capa de lógica del negocio posee el controlador. Finalmente, la capa de datos o de persistencia almacena la información referente a los datos del negocio (las variables de los sensores). Para tener una arquitectura y diseño solido se usa un marco de trabajo o framework llamado Laravel basado en MVC. Por último, la persistencia se puede obtener por medio de una base de datos estructurada entre Laravel y MySQL, por ejemplo.

\subsection{Diseño gráfico y funcional}

Al ser una aplicación web, la funcionalidad de la interfaz deriva de su facilidad de consultar información mediante un navegador web (en smartphones, tablet, portátil o PC) conectado al servidor, sin tener que instalar el software (lo que ocurriría con un software de escritorio). Para construir la interfaz gráfica se utilizó Bootstrap, este framework nos proporciona un "cascaron " con un diseño profesional y editable, sin tener que lidiar con el diseño de un sitio, sobre el cual se construyó la interfaz web de la estación terrena, además ofrece la ventaja del ahorro de tiempo para integrar la información ya que este cuenta con una estructura definida y esta optimizado para ser responsivo. 


\section{Resultados y conclusiones}

Se cumplió con los objetivos, obteniéndose un sistema integral de bajo costo, que además ofrece la ventaja de ser un sistema con portabilidad y al mismo tiempo escalable. Este software deja un precedente de como hacer software de estaciones terrenas para este tipo de concursos. Se obtiene un circuito encargado de transmitir información de las diferentes variables que se necesitaban cubrir, con un peso 165 gramos de carga total. En la figura 15 se presenta el circuito encargado de la telemetría.

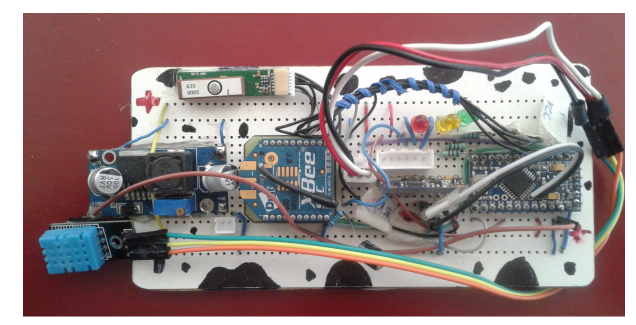

Fig. 15. Circuito de telemetría.

Asimismo, durante el concurso el proceso de medición funcionó correctamente. Aunado a esto el software fue la mejor estación terrena. Lo que en consecuencia se logrará tener un primer lugar en el concurso. Por otra parte, La interfaz del sistema de monitoreo, permitió la manipulación de la estación por parte de otros usuarios de manera sencilla, a la vez que permitió la comunicación con computadoras, por ejemplo en el caso de la geolocalización, en el cual los jueces podían verificar, en tiempo real, la ubicación a través del API embedido de Google Maps.

Finalmente, los resultados permiten concluir que es posible desarrollar estaciones de monitoreo o estaciones terrenas de bajo costo, a partir de dispositivos electrónicos y software de uso común, con rendimiento similar al de los equipos comerciales. Sin embargo, hay que ser conscientes de que este tipo de desarrollos depende siempre de variables como: internet, dominio o ambiente, infraestructura, entre otras cosas. A pesar de esto, se puede decir que la estación terrena es un sistema integral que podría reducir considerablemente los costos en un proyecto real en el tema de la tecnología espacial o de meteorología, por ejemplo.

En la figura 16 se muestra la vista del diseño gráfico de la interfaz para la estación terrena.

Agradecimientos. Este trabajo ha sido apoyado por el Instituto Politécnico Nacional, México (IPN) y el Centro de Desarrollo Aeroespacial (CDA) del IPN. Además este trabajo es desarrollado con recursos e instalaciones del IPN. Cabe resaltar que los resultados de este trabajo fueron realizados por el tesista de Nivel 
Software de estación terrena para cohetes hidropropulsados
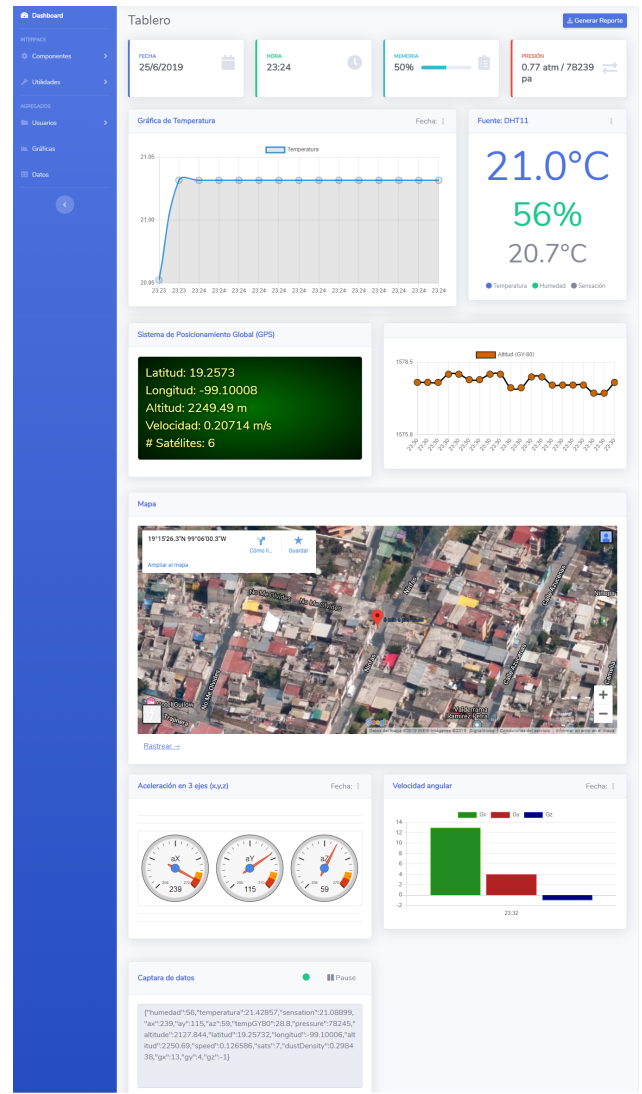

Fig. 16. Vista de la interfaz terrena.

Maestría Alberto Carlos Pérez Cadena. También, se le agradece a los revisores que aportaron sus valiosos conocimientos para mejora del presente artículo.

\section{Referencias}

1. Estructura y control térmico de nanosatélites (Diciembre 2013)

2. Agencia Espacial Mexicana: Ingeniería de sistehttps://www.educacionespacial.aem.gob.mx/images_ise/pdf/01introduccion.pdf/ (2017), consultado el 22-04-2019

3. Bertoglio, O.J., Johansen, O.: Introducción a la teoría general de sistemas. Editorial limusa (1982)

4. Heath, S.: Embedded systems design. Elsevier (2002)

5. LaCombe, J.C., Wang, E.L., Nicolescu, M., Rivera, P., Poe, B.: Design experiences with a student satellite program. In: Proceedings of the 2007 American Society for Engineering Education Pacific Southwest Annual Conference (2007) 
6. Larson, W.J., Wertz, J.R.: Space mission analysis and design. Tech. rep., Torrance, CA (United States); Microcosm, Inc. (1992)

7. Luján-Mora, S.: Programación de aplicaciones web: historia, principios básicos y clientes web. Editorial Club Universitario (2002)

8. Pérez, P.D.: Sistema embebido basado en el monitoreo de consumo de energía eléctrica de electrodomésticos para el control del gasto. Revista Tecnología Digital. Vol 8(1), 69-80 (2018)

9. Rivera, A.F.G., Clavijo, F.V.: Monitoreo de sensores en aplicaciones web embebidas. In: International Congress of Basic Sciences and Engineering CICI. pp. 4399-4402. No. 105 (2016)

10. Ruiz-Ayala, D.C., Vides-Herrera, C.A., Pardo-García, A.: Monitoreo de variables meteorológicas a través de un sistema inalámbrico de adquisición de datos. Revista de investigación, Desarrollo e Innovación 8(2), 333-341 (2018)

11. Sommerville, I., Galipienso, M.I.A.: Ingeniería del software. Pearson Educación (2005)

12. Stair, R., Moisiadis, F., Genrich, R., Reynolds, G.: Principles of information systems: A managerial approach (2010)

13. Vela, R.N.: Comunicaciones por Satélite. México. International Thomson Editores (2003) 